\title{
Underlying the Factors of Unmet Need for Family Planning in Indonesia: A Spatial Analysis
}

\author{
Ardiana Irma ${ }^{1} \&$ Ekoriano Mario ${ }^{1}$ \\ ${ }^{1}$ National Population and Family Planning Board, Jakarta Timur, Indonesia \\ Correspondence: Ekoriano Mario, National Population and Family Planning Board, Jl. Permata No.1 \\ Halimperdanakusuma, Jakarta Timur, 13650, Indonesia. Tel: 62-812-9171-2229.
}

$\begin{aligned} & \text { Received: October 19, } 2020 \quad \text { Accepted: December 7, } 2020 \quad \text { Online Published: December 18, } 2020 \\ & \text { doi:10.5539/gjhs.v13n2p6 }\end{aligned}$ URL: https://doi.org/10.5539/gjhs.v13n2p6

\begin{abstract}
Unmet need for family planning remains a challenge in Indonesia. The Government has incorporated into the 2020-2024 National Medium-term Development Plan, an ambitious target to reduce unmet need from 12,4\% in 2018 to $7.4 \%$ in 2024 . This will require significant effort, while systematic and updated studies explaining the underlying causes are scarce, especially those that look at the level within the diverse characteristics of Indonesia. This study therefore aimed to investigate the fundamental factors contributing to unmet need for family planning, categorized into five dimensions (D): D.1). weak or ambivalent fertility preferences; D.2). generic disapproval of pregnancy prevention; D.3). method-specific barriers to use; D.4). perceived low risk of getting pregnant; and D.5). partner-related factors. This study also mapped the pattern at the sub-national level namely Java-Bali (region 1/R1), outside Java-Bali 1 (region 2/R2) and outside Java-Bali 2 (region 3/R3). Findings show that the level of unmet need is highest in R3 which consists of provinces that started implementing their family planning program much later than provinces in the other two regions. The study also shows that women between 20-29 years old, with educated husband and larger family size, who consider smaller family size as ideal, are more likely to have unmet need compared to their counterparts in all regions. The likelihood of unmet need is most prominent in the region with the most mature program. The study emphasizes the importance of designing appropriate targeted interventions that are tailored to the local context and not apply a 'one-size-fits-all' policy.
\end{abstract}

Keywords: unmet need, contraceptive, family planning

\section{Introduction}

To continue the momentum generated by the Millennium Development Goals (MDGs), world leaders agreed to consolidate and make all the achievements more sustainable by launching the Sustainable Development Goals (SDGs). This global agenda places comprehensive and inclusive health in a central position as stated in Goal 3 that calls for actions to "ensure healthy lives and promote well-being for all at all ages", by 2030. Target 3.7 aims to "ensure universal access to sexual and reproductive health-care services, including for family planning, information and education, and the integration of reproductive health into national strategies and programs." The family planning indicator for this target is the, "proportion of women of reproductive age (aged 15-49 years) who have their need for family planning satisfied with modern methods" (Ministry of National Development Planning/National Development Planning Agency, 2020). This commitment and the International Conference on Population and Development (ICPD) Program of Action, Nairobi Summit 2019, put emphasis on achieving universal access to sexual and reproductive health and rights as part of universal health coverage. Ending unmet need for family planning information and services as well as ensuring universal availability of quality, accessible, affordable and safe modem contraceptives, is one concrete commitment that can be made to ensure full, effective and accelerated implementation of the ICPD Program of Action and the SDGs Agenda.

Many countries, including Indonesia, as a member of the global community, are committed to growing and improving the voluntary family planning program. Family planning is also included in Indonesia's 2020-2024 National Medium-Term Development Plan as one strategy to improve health service provision and achieve universal health coverage. Unmet need for family planning is a negative indicator of access and quality of family planning service, which in the last four years (2015-2018) in Indonesia, has shown a fluctuating trend and recently experienced a plateau 
(Figure 1). Between 2015 and 2018, Indonesia has succeeded to decrease unmet need by only 2 percent (National Population and Family Planning Board, 2019). There are also wide disparities in unmet need between provinces, from as high as 23.2 percent in East Nusa Tenggara to as low as 4.9 percent in South Kalimantan (Figure 2) (National Population and Family Planning Board, 2018).

An abundance of research has shown that low access to family planning services is strongly associated with unintended pregnancies and adverse maternal health outcomes (Bishwajit, Tang, Yaya, \& Feng, 2017; Bellizzi, Mannava, Nagai, \& Sobel, 2020; WHO, 2019). Improving access to contraceptives is therefore one of the Government of Indonesia's commitments. Through increased investment and effort, the national family planning program is looking to close the gap and is targeting to reduce the unmet need for family planning to 7.4 percent by year 2024 (National Population and Family Planning Board, 2020).

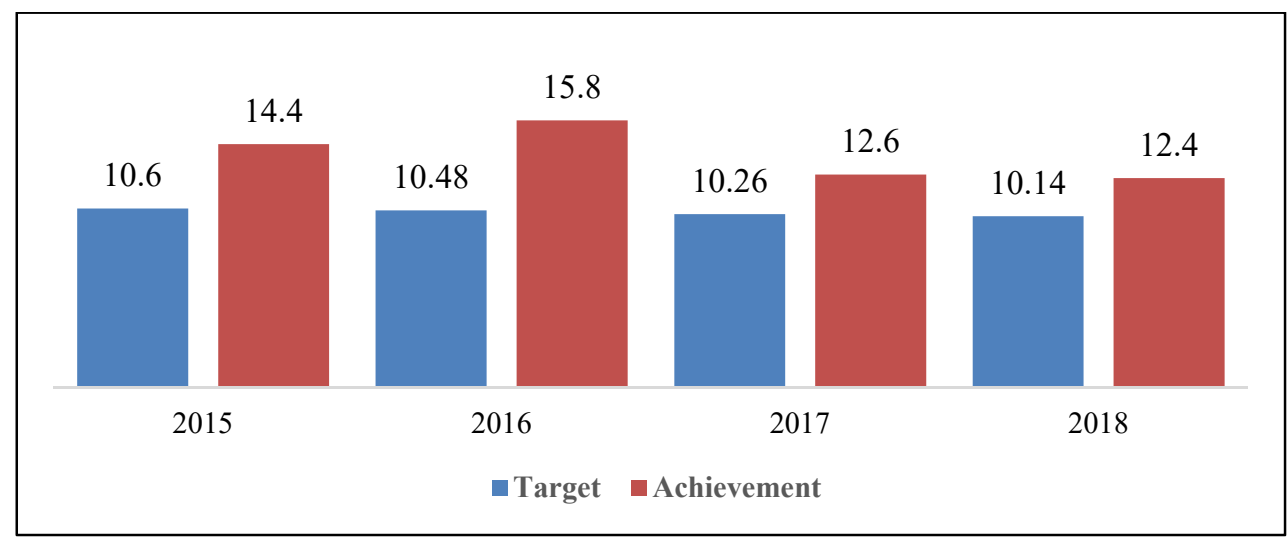

Figure 1. Target and achievement of unmet need for family planning in Indonesia: 2015-2018

Source: National Population and Family Planning Board, 2019

In the absence of a comprehensive and inclusive strategy, addressing the unfulfilled demand for contraception, and fully implementing the ICPD program of action will be a difficult, if not impossible, task. This study therefore aimed to formulate recommendations for policy development that does not rely on a 'one size fits all' approach. This is a highly relevant concept for Indonesia with its geographic and cultural diversity, varying levels of family planning program maturity, as well as different optimization of available resources. The study carefully examined current fundamental factors that contribute to the unmet need for family planning and used a causal framework introduced by Machiyama et al., 2017 to explain how those factors prevent individuals from accessing contraceptive services. The framework categorizes the factors into five dimensions: 1). weak fertility preferences (dimension 1/D1); 2). generic disapproval of pregnancy prevention (dimension 2/D2); 3). method-specific barriers to use (dimension 3/D3); 4). perceived low risk of getting pregnant (dimension 4/D4); and 5). partner-related factors (dimension 5/D5). 


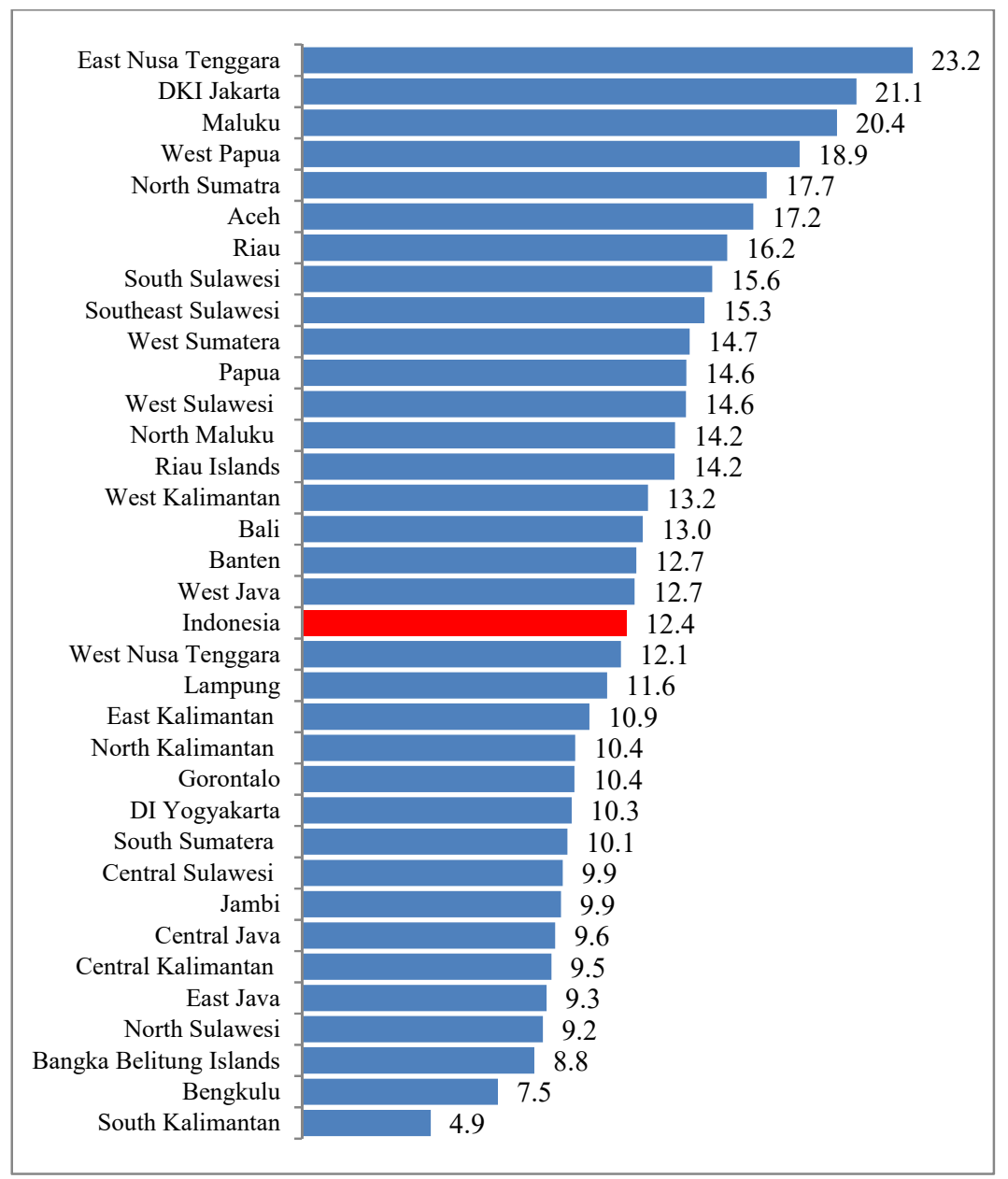

Figure 2. Percentage of unmet need by province, 2018

Source: National Population and Family Planning Board, 2018

\section{Methodology}

\subsection{Material Studied}

The study used secondary data from the 2018 Government Performance and Accountability Survey (GPAS), an annual national survey conducted by the National Population and Family Planning Board (or Badan Kependudukan dan Keluarga Berencana Nasional/BKKBN) with a goal to measure the performance of population, family planning and family development indicators encapsulated in the 2015-2019 Strategic Plan of the National Population and Family Planning Board and the 2015-2019 National Medium-Term Development Plan. The survey is designed to provide representative estimate of the indicators at both the national and provincial level.

\subsection{Area Description}

In the GPAS survey, the 34 provinces of Indonesia were grouped into regions following a classification procedure that was established by the Government of Indonesia in 1969 and is still relevant today (Soesastro et al., 2005). Provinces were grouped on the basis of their population density and the urgency for implementing a family planning program. Provinces in the 'Java-Bali' region (region 1/R1) were the first to implement the family planning program in Indonesia's first five-year development plan (1969-1974), followed by provinces 'outside Java Bali 1' (region 2/R2) and 'outside Java Bali 2' (region 3/R3) in subsequent two cycles of five-year development plans. Distribution of provinces and regions is illustrated in Figure 3. 


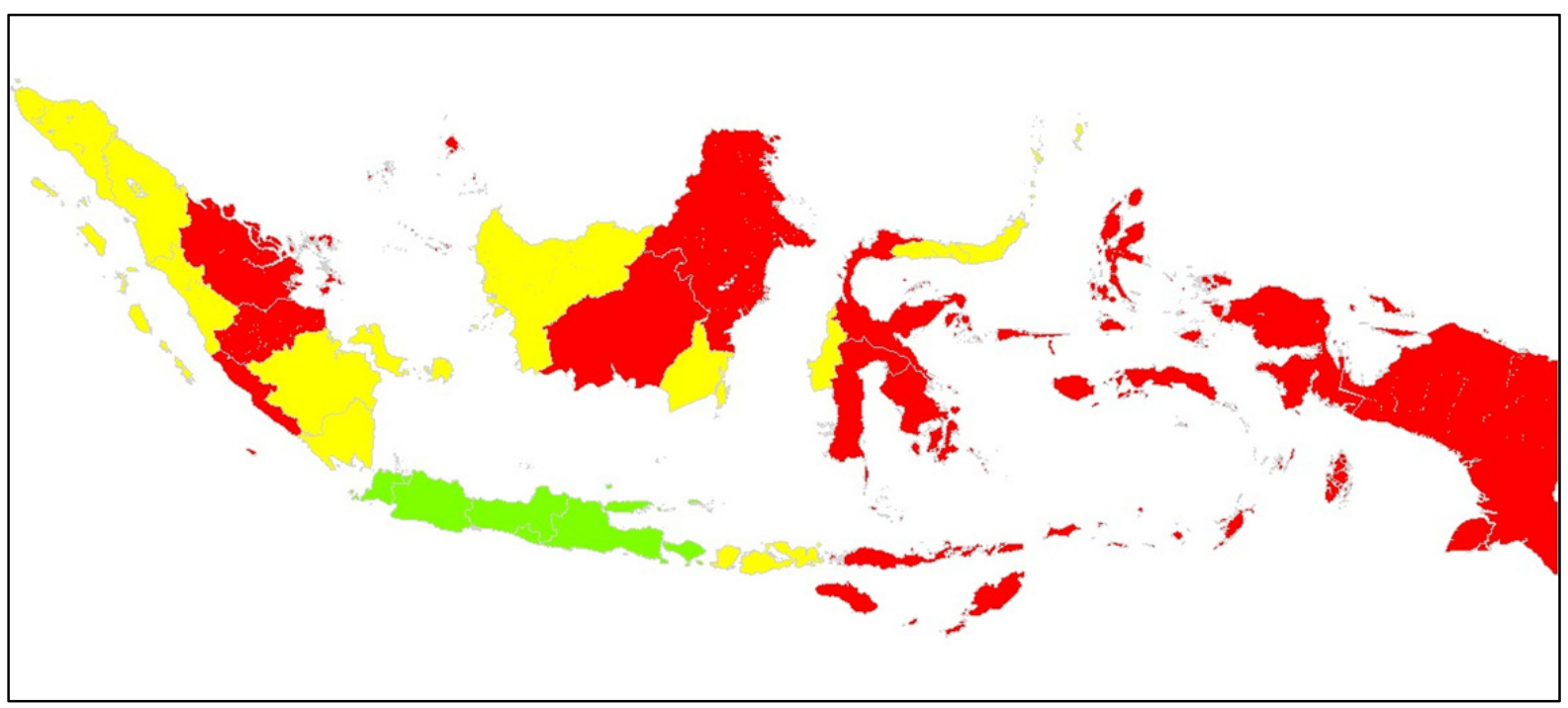

Figure 3. Distribution of Provinces by Regions

Region Java-Bali/Region 1/R1

Region Outside Java-Bali 1/Region 2/R2

Region Outside Java-Bali 2/Region 3/R3

\subsection{Methods and Techniques}

The survey utilized three sampling frameworks. The first stage utilized a list of villages/urban villages throughout Indonesia, complete with their urban/rural classification. In addition, wealth index was also considered during selection of villages/urban villages. The second sampling framework generated a list of clusters in selected villages/urban villages, while the third stage of sampling selected households based on household listings that were generated by enumerators who went door-to-door in selected clusters.

Survey's enumeration areas were clusters which consisted of several nearby census blocks and contained approximately 200 households. Stratified multistage random sampling was used as the overall sampling strategy. A total of 1,935 villages in Indonesia (spread across 34 provinces and 514 districts) were selected as clusters using a probability proportionate to size sampling method based on the socio-economic background (rural-urban and wealth index) of different areas. Villages/urban villages were selected independent of their urban/rural category. Subsequently, 35 households were selected from each cluster using systematic random sampling performed door to door by enumerators/interviewers in selected clusters, yielding a total of 67,725 households.

Pretesting of the questionnaire was performed prior to data collection to assess the extent respondents are able to answer the list of questions, and to observe how the questions fit into the smartphone application. Household listing practice was performed during the trial to know the number of households in the selected clusters that could be used as survey samples. The listing practice also intended to better understand the mechanism of listing and the number of interviewers that would be ideal to conduct listing in the field. Pretesting and trial were carried out in March 2019 in Depok Sub-District, Depok City, West Java. Interviewers went door-to-door in the selected cluster.

The GPAS has 4 (four) modules: household, family, women of reproductive age (15-49 years) and unmarried adolescents between the age of 15-24. The survey used the current legal definition of a family i.e. the smallest social unit of society, that can be a husband and a wife, a husband, a wife and their children, a father with his children or a mother with her children. The household module is the gateway to the other series of modules. It identifies other relevant respondents through listed members of the household. Survey data was collected digitally to ensure quality and facilitate quick analysis of data. Enumerators were equipped with a mobile phone and an application which includes all the modules, digitized and connected.

This study used the household and the women of reproductive age (15-49 years) modules. The women of reproductive age (15-49 years) module contained the following variables: sex, wife's occupation, husband/spouse's occupation, 
family relationship, marital status, wealth index and spousal age. The unit of analysis focused on 47,053 married women, 18,654 of whom were not using any modern contraceptive method. Among the non-contraceptive users, 2,117 women were pregnant or in the post-partum amenorrhea period, and among them, 329 women experienced an unmet need for either spacing (235) or limiting (94) births. Region-wise, 150, 58 and 27 women in R1, R2 and R3 regions consecutively had an unmet need for spacing while 63,18 and 13 women in R1, R2 and R3 regions consecutively had an unmet need for limiting. Among the rest of non-contraceptive users who were neither pregnant nor postpartum amenorrheic, 3,577 women experienced an unmet need for limiting births, namely 2,118 women in R1; 923 in R2; and 527 in R3.

Data was analyzed using descriptive and inferential analysis. The descriptive analysis portrayed the distribution of selected socio-demographic variables both for spacers and limiters by regions. This analysis also explored some additional background characteristics to gain insights about the unmet need in each region. The underlying causes of unmet need in all 34 provinces are visualized in a quadrant map that identifies each province's position relative to the dimensions of unmet need (see appendix). In the inferential analysis, regression logistics was used to investigate the predictive power of selected independent variables including all dimensions on unmet need. It captured the dynamic relationship in three models i.e Java-Bali, outside Java-Bali 1 and outside Java-Bali 2 . This allows one to identify the independent variables that have the greatest effect on the dependent variables in each area/region. Variables with the greatest effect can be a basis for setting policy priorities in the family planning program.

\subsection{Concept of Unmet Need for Family Planning}

The GPAS uses the definition and widely accepted method by Bradley to measure unmet need for family planning (Bradley et al., 2012).

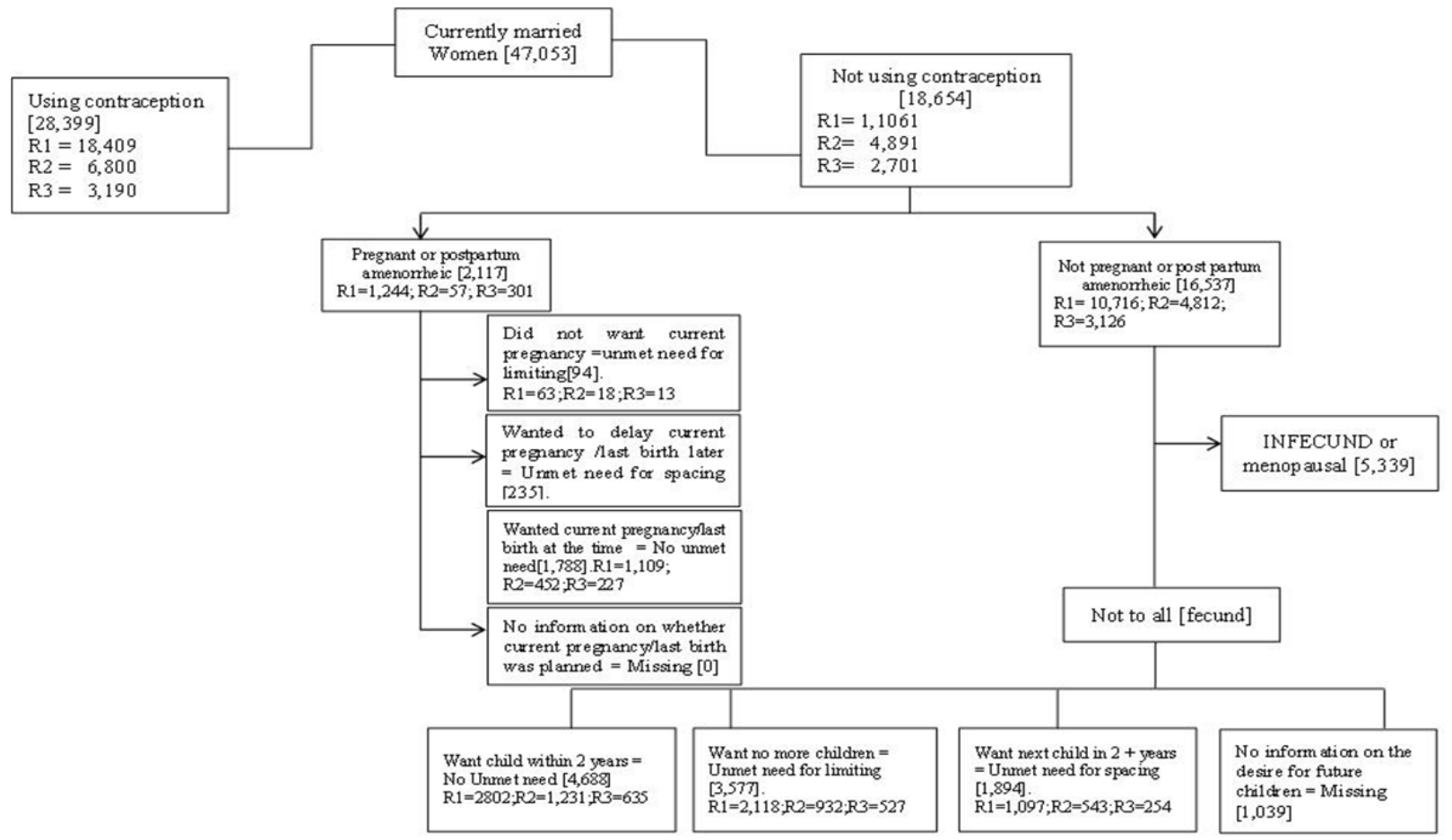

Figure 3. Unit of analysis

Source: processed data.

In the survey questionnaire asked about, there are 24 possible answers provided to the question asking the 'reason' that women who want to limit or postpone their subsequent child but yet did not practice any modern contraceptive method. Respondents were provided with 24 possible answers and were allowed to choose more than one answer. The list of possible answers included: 1). unmarried/not yet married; 2). less frequent sexual intercourse/far distance husband; 3). 
already menopausal/has had hysterectomy; 4). infertile/less fertile; 5). in post-partum amenorrhea period; 6). breastfeeding; 7). husband has left for several days; 8). it is up to God/fatalistic; 9). respondent's disapproval of contraceptives; 10). husband's/spousal disapproval; 11). other family member's disapproval; 12). religious prohibition; 13). uninformed about family planning methods; 14). uninformed about health facility that provides family planning service; 15). fear of contraceptive side effects; 16). health concern; 17). limited access to/unreachable health facility; 18). too expensive; 19). unavailability of preferred method; 20). unavailability of any contraceptive services; 21). feel uncomfortable; 22). body weight changes; 23). other reasons; 24). do not know. Based on the above options, except for the answers 23 and 24, the causal framework was adopted to simplify the reasons for not using any contraceptive. The answers were categorized into the corresponding dimensions as follows (Machiyama et al., 2017).

Table 1. Dimensions of unmet need

\begin{tabular}{ll}
\hline Dimensions & Answers \\
\hline Weak fertility preferences & 8). it is up to God/fatalistic \\
\hline Generic disapproval of pregnancy prevention & 9). respondent's disapproval; \\
\hline & 11). other family member's disapproval; \\
& 12). religious prohibition \\
\hline 13). uninformed about family planning methods; & 14). uninformed about health facility that provides family \\
planning service; & 15). fear of contraceptive side effects; \\
& 16). health concern; \\
& 17). limited access to/unreachable health facility; \\
& 18). too expensive; \\
& 19). unavailability of preferred method; \\
& 20). unavailability of any contraceptive services; \\
& 21). feel uncomfortable; \\
22). body weight changes
\end{tabular}

1). unmarried/not yet married;

2). less frequent sexual intercourse/far distance husband;

3). already menopausal/has had hysterectomy;

Perceived low risk of getting pregnant

4). infertile/less fertile;

5). in post-partum amenorrhea period;

6). breastfeeding;

7). husband has left for several days

Partner-related factors 10 ). husband's/spousal disapproval

Source: Machiyama et al., 2017 \& processed data.

\subsection{Data Management}

Data management in all 34 provinces followed a structure that was established to ensure consistent data quality from enumerators to data managers and from data collection to dissemination. All personnel who were involved in the data process received a 14-day training. Data management was organized as follows:

a. Data Management Team. Researchers at the Central BKKBN Office were assigned to be in charge of a Data Management Team. This team was responsible for ensuring that the process of listing, data collection, and 
re-interview (if necessary) proceeded smoothly, and that data received from enumerators were verified and validated before being uploaded to the server. Cleaned data was subsequently sent to Statistics Indonesia to be weighted accordingly, allowing an estimation of measures at the national and provincial level. The Data Management Team also attended a 7-day "Training of Master Trainers" designed to equip them to train facilitators and supervisors in follow-on trainings.

b. Facilitators. Facilitators were appointed at the provincial level and functioned as a link between the data management team and provincial supervisors. Facilitators were BKKBN's researchers, computer experts and personnel from designated local universities. University representatives were lecturers with a graduate degree and experience in field survey and/or research.

c. Supervisors. Supervisors were recruited from a local university with a minimum accreditation level B. They supervised the data collection process from listing to interview.

d. Enumerators. Enumerators were tasked with face-to-face interviews using a structured questionnaire, and completing the questionnaire through enumerators' mobile phone. Digital data collection enabled data to be sent directly to the server. Enumerators had to have at least a Diploma 1 degree (High School +1 year). Their numbers varied between provinces depending on the size of the clusters, and each enumerator was in charge of maximum 3 (three) clusters.

\subsection{Ethical Clearence}

The study received approval from an Ethical Review Team through a letter from the Head of the Ethical Team number 1281/PD.101/H4/2018. Survey implementation was based on the regulation of BKKBN's Chairman number 11/2018.

\section{Results}

\subsection{Descriptive Analysis}

In all 3 regions, the unmet need for limiting childbirth was found to be higher than that for spacing births. Among the three regions, provinces outside Java-Bali 2 (Region 3/R3) had the highest unmet need at 14 percent, closely followed by R2 at 13.3 percent. The lowest level of unmet need was found in R1 at 11.7 percent (Table 2). Unmet need for family planning seems to be associated with women who live in urban areas, are older, have higher education, and married someone with a higher level of education as well. These women also tend to regard a larger family size as ideal and bear three or more children. A look at the correlation between wealth index and unmet need showed an uneven pattern. In R1 and R3, unmet need seems to directly correlate with family income level, but in R3 unmet need was concentrated among the poorest women. Data on selected demographic indicators in each region shown in Table 3 indicates that R1 has a more advanced family planning program and better economic status compared to the other two regions. 
Table 2. Distribution of Unmet Need by Demographic Characteristics and Region

\begin{tabular}{|c|c|c|c|c|c|c|c|c|c|c|c|c|c|}
\hline \multirow[b]{2}{*}{ Variables } & \multirow[b]{2}{*}{ Categories } & \multicolumn{4}{|c|}{ Classification [Java Bali] } & \multicolumn{4}{|c|}{ Classification [Outside Java Bali 1] } & \multicolumn{4}{|c|}{ Classification [Outside Java Bali 2] } \\
\hline & & $\begin{array}{c}\text { Unmet need } \\
\text { for spacing } \\
\text { Percent }\end{array}$ & $\begin{array}{c}\text { Unmet need } \\
\text { for limiting } \\
\text { Percent }\end{array}$ & $\begin{array}{c}\text { Unmet need } \\
\text { Percent }\end{array}$ & Count & \begin{tabular}{|c|} 
Unmet \\
need for \\
spacing \\
Percent
\end{tabular} & $\begin{array}{c}\text { Unmet } \\
\text { need for } \\
\text { limiting } \\
\text { Percent }\end{array}$ & $\begin{array}{c}\text { Unmet need } \\
\text { Percent }\end{array}$ & Count & \begin{tabular}{|c} 
Unmet \\
need for \\
spacing \\
Percent
\end{tabular} & $\begin{array}{c}\text { Unmet } \\
\text { need for } \\
\text { limiting } \\
\text { Percent }\end{array}$ & $\begin{array}{c}\text { Unmet need } \\
\text { Percent }\end{array}$ & Count \\
\hline \multirow{2}{*}{ Residence } & Urban & 4.8 & 8.8 & 13.6 & 2302 & 5.7 & 9.5 & 15.2 & 600 & 4.8 & 11.1 & 15.9 & 311 \\
\hline & Rural & 3.5 & 5.6 & 9.0 & 1126 & 4.9 & 7.5 & 12.3 & 951 & 4.8 & 8.2 & 13.0 & 511 \\
\hline Total & & 4.2 & 7.4 & 11.7 & 3428 & 5.2 & 8.1 & 13.3 & 1551 & 4.8 & 9.2 & 14.0 & 822 \\
\hline \multirow{7}{*}{ Age groups } & $15-19$ & 7.4 & 0.4 & 7.7 & 28 & 13.3 & 0.5 & 13.7 & 27 & $\overline{c 10.3}$ & 0.3 & 10.5 & 10 \\
\hline & $20-24$ & 8.6 & 1.0 & 9.6 & 239 & 11.1 & 1.5 & 12.6 & 121 & 10.3 & 0.8 & 11.2 & 56 \\
\hline & $25-29$ & 9.3 & 2.1 & 11.4 & 549 & 9.8 & 2.8 & 12.7 & 237 & 10.6 & 3.8 & 14.3 & 134 \\
\hline & $30-34$ & 5.1 & 4.6 & 9.7 & 528 & 6.7 & 5.4 & 12.1 & 286 & 5.4 & 6.1 & 11.5 & 137 \\
\hline & $35-39$ & 2.9 & 7.1 & 9.9 & 577 & 3.5 & 7.5 & 11.1 & 273 & 3.3 & 10.1 & 13.5 & 171 \\
\hline & $40-44$ & 1.1 & 11.4 & 12.5 & 704 & 1.4 & 14.4 & 15.8 & 343 & 1.1 & 14.3 & 15.5 & 164 \\
\hline & $45-49$ & 1.1 & 15.5 & 16.6 & 801 & 0.6 & 15.6 & 16.2 & 265 & 0.3 & 17.8 & 18.1 & 151 \\
\hline Total & & 4.2 & 7.4 & 11.7 & $\begin{array}{l}3428 \\
\end{array}$ & 5.2 & 8.1 & 13.3 & 1551 & 4.8 & 9.2 & 14.0 & 822 \\
\hline \multirow{3}{*}{$\begin{array}{l}\text { Level of education } \\
\text { (husband) }\end{array}$} & Low & 2.5 & 6.9 & 9.5 & 1075 & 4.1 & 7.3 & 11.4 & 489 & 4.1 & 8.4 & 12.5 & 253 \\
\hline & Middle & 4.4 & 6.0 & 10.3 & 671 & 4.6 & 7.3 & 11.9 & 325 & 3.9 & 8.5 & 12.4 & 159 \\
\hline & above & 6.1 & 7.3 & 13.3 & 1681 & 6.5 & 7.5 & 14.1 & 737 & 5.8 & 9.2 & 15.0 & 409 \\
\hline Total & & 4.5 & 7.1 & 11.7 & 3428 & 5.5 & 7.8 & 13.3 & 1551 & 5.0 & 9.0 & 14.0 & 822 \\
\hline \multirow{3}{*}{ Level of education (wife) } & Low & 2.6 & 8.5 & 11.2 & 1175 & 3.1 & 8.6 & 111.7 & 479 & 3.2 & 9.5 & 12.7 & 254 \\
\hline & Middle & 4.1 & 5.9 & 10.0 & 748 & 5.0 & 8.5 & 13.4 & 368 & 4.9 & 8.5 & 13.5 & 176 \\
\hline & Above & 5.8 & 7.4 & 13.2 & 1505 & 7.0 & 7.5 & 14.6 & 704 & 6.0 & 9.2 & 15.2 & 391 \\
\hline Total & & 4.2 & 7.4 & 11.7 & 3428 & 5.2 & 8.1 & \begin{tabular}{|l|}
13.3 \\
\end{tabular} & 1551 & 4.8 & 9.2 & 14.0 & 822 \\
\hline \multirow{2}{*}{ Ideal family size } & $0-2$ & 3.5 & 7.7 & 11.2 & 2242 & 4.8 & 7.2 & 11.9 & 722 & 4.9 & 8.6 & 13.5 & 415 \\
\hline & $>=3$ & 5.8 & 6.8 & 12.6 & 1186 & 5.6 & 9.2 & 14.8 & 829 & 4.6 & 9.9 & 14.5 & 407 \\
\hline Total & & 4.3 & 7.4 & 11.7 & 3428 & 5.2 & 8.1 & 13.3 & 1551 & 4.8 & 9.2 & 14.0 & 822 \\
\hline \multirow{2}{*}{ Children ever born (CEB) } & $0-2$ & 5.1 & 6.0 & 11.1 & 2441 & 7.1 & 4.5 & 11.6 & 853 & 6.6 & 5.4 & 12.0 & 423 \\
\hline & $>=3$ & 1.7 & 11.6 & 13.3 & 987 & 1.9 & 14.4 & 16.3 & 699 & 2.1 & 14.9 & 17.0 & 398 \\
\hline Total & & 4.3 & 7.4 & 11.7 & 3428 & 5.2 & 8.1 & 13.3 & 1551 & 4.8 & 9.2 & 14.0 & 822 \\
\hline \multirow{5}{*}{ Wealth index } & Lowest & 3.3 & 6.5 & 9.8 & 319 & 5.4 & 7.7 & 13.1 & 392 & 6.5 & 8.8 & 15.3 & 315 \\
\hline & Middle below & 3.2 & 7.7 & 10.9 & 553 & 5.2 & 7.6 & 12.8 & 359 & 3.9 & 8.9 & 12.9 & 147 \\
\hline & Middle & 4.3 & 7.2 & 11.5 & 714 & 5.0 & 8.0 & 13.1 & 323 & 4.2 & 7.9 & 12.1 & 113 \\
\hline & Middle above & 5.1 & 6.8 & 11.9 & 822 & 5.1 & 9.6 & 14.7 & 306 & 4.1 & 9.5 & 13.6 & 135 \\
\hline & above & 4.5 & 8.3 & 12.9 & 1019 & 4.8 & 8.2 & 13.0 & 172 & 3.1 & 11.9 & 14.9 & 111 \\
\hline Total & & 4.2 & 7.4 & 11.7 & 3428 & 5.2 & 8.1 & 13.3 & 1551 & 4.8 & 9.2 & 14.0 & 822 \\
\hline
\end{tabular}

Source: processed data 
Table 3. Selected Demographic Characteristics by Region

\begin{tabular}{|c|c|c|c|c|c|c|c|c|c|c|c|}
\hline \multirow{3}{*}{ Province/Region } & \multirow[b]{2}{*}{ Ideal Family Size } & \multirow[b]{2}{*}{ CEB } & \multicolumn{4}{|c|}{ Wealth index } & \multirow[b]{2}{*}{ Highest } & \multicolumn{3}{|l|}{ Contraceptive use } & \multirow{3}{*}{$\begin{array}{l}\text { Total } \\
\text { percent }\end{array}$} \\
\hline & & & Lowest & Middle low & Middle & Middle above & & $\begin{array}{l}\text { Not using } \\
\text { contraceptive }\end{array}$ & Traditional & Modern & \\
\hline & Mean & Mean & percent & percent & percent & percent & percent & percent & Percent & Percent & \\
\hline Jakarta & 2.4 & 1.8 & 3.3 & 3.8 & 12.4 & 31.7 & 48.8 & 57.2 & 2.1 & 40.7 & 100 \\
\hline West Java & 2.5 & 2.1 & 15.7 & 19.3 & 20.6 & 21.6 & 22.9 & 39.3 & 1.6 & 59.1 & 100 \\
\hline Central Java & 2.2 & 2.0 & 11.3 & 19.1 & 21.4 & 22.2 & 26.0 & 32.6 & 4.8 & 62.6 & 100 \\
\hline Yogyakarta & 2.2 & 1.8 & 5.4 & 13.4 & 28.2 & 22.9 & 30.1 & 35.0 & 11.2 & 53.9 & 100 \\
\hline East Java & 2.3 & 1.8 & 7.8 & 17.8 & 24.4 & 25.3 & 24.7 & 32.1 & 4.8 & 63.1 & 100 \\
\hline Banten & 2.5 & 1.9 & 10.1 & 14.8 & 20.0 & 24.3 & 30.8 & 45.3 & 1.9 & 52.7 & 100 \\
\hline Bali & 2.4 & 2.1 & 7.7 & 18.6 & 16.6 & 19.0 & 38.2 & 37.1 & 4.9 & 58.0 & 100 \\
\hline R1 [Java Bali] & 2.4 & 2.0 & 11.0 & 17.3 & 21.2 & 23.5 & 27.0 & 37.5 & 3.5 & 59.0 & 100 \\
\hline Aceh & 3.4 & 2.5 & 17.5 & 23.4 & 25.0 & 20.5 & 13.7 & 53.6 & 2.4 & 44.1 & 100 \\
\hline North Sumatera & 3.0 & 2.6 & 24.9 & 19.9 & 19.6 & 23.1 & 12.5 & 45.8 & 7.5 & 46.7 & 100 \\
\hline West Sumatera & 2.8 & 2.3 & 13.7 & 21.9 & 26.4 & 23.4 & 14.6 & 48.0 & 3.7 & 48.3 & 100 \\
\hline South Sumatera & 2.6 & 2.1 & 31.0 & 26.0 & 20.8 & 12.9 & 9.3 & 42.9 & 0.7 & 56.4 & 100 \\
\hline Lampung & 2.6 & 2.1 & 15.8 & 27.7 & 27.9 & 18.7 & 10.0 & 33.3 & 2.7 & 64.1 & 100 \\
\hline Bangka Belitong island & 2.5 & 2.1 & 4.2 & 11.7 & 25.8 & 34.2 & 24.1 & 31.5 & 3.9 & 64.5 & 100 \\
\hline West Nusa Tenggara & 2.9 & 2.2 & 18.7 & 28.6 & 27.7 & 14.7 & 10.4 & 40.6 & 1.6 & 57.8 & 100 \\
\hline West Kalimantan & 2.7 & 2.3 & 52.3 & 23.0 & 13.5 & 6.5 & 4.6 & 37.7 & 1.9 & 60.3 & 100 \\
\hline South Kalimantan & 2.2 & 1.7 & 32.2 & 32.0 & 8.0 & 16.5 & 11.2 & 36.2 & 0.6 & 63.2 & 100 \\
\hline North Sulawesi & 2.2 & 2.0 & 25.0 & 27.2 & 21.0 & 18.5 & 8.3 & 34.5 & 1.1 & 64.4 & 100 \\
\hline South Sulawesi & 2.7 & 2.4 & 34.0 & 19.8 & 16.1 & 17.2 & 13.0 & 43.3 & 4.0 & 52.7 & 100 \\
\hline Gorontalo & 2.4 & 2.4 & 23.7 & 24.4 & 19.0 & 19.1 & 13.8 & 36.3 & 2.5 & 61.2 & 100 \\
\hline West Sulawesi & 2.9 & 2.7 & 42.9 & 25.2 & 13.6 & 10.1 & 8.2 & 50.8 & 2.9 & 46.3 & 100 \\
\hline R2 [Outside Java Bali 1] & 2.7 & 2.3 & 25.7 & 24.0 & 21.2 & 17.9 & 11.3 & 41.8 & 3.2 & 54.9 & 100 \\
\hline
\end{tabular}




\begin{tabular}{|c|c|c|c|c|c|c|c|c|c|c|c|}
\hline Riau & 2.8 & 2.4 & 19.2 & 13.3 & 17.3 & 27.6 & 22.7 & 43.6 & 4.2 & 52.2 & 100 \\
\hline Jambi & 2.5 & 2.2 & 22.2 & 20.4 & 18.7 & 20.7 & 18.0 & 38.2 & 3.9 & 57.9 & 100 \\
\hline Bengkulu & 2.4 & 2.2 & 11.0 & 23.3 & 29.9 & 23.6 & 12.2 & 31.7 & 3.7 & 64.6 & 100 \\
\hline Riau Island & 2.6 & 2.1 & 16.8 & 18.9 & 27.3 & 24.4 & 12.7 & 56.3 & 0.6 & 43.1 & 100 \\
\hline East Nusa Tenggara & 3.0 & 2.8 & 80.2 & 12.1 & 3.7 & 2.2 & 1.9 & 57.6 & 4.0 & 38.4 & 100 \\
\hline Central Kalimantan & 2.6 & 2.2 & 51.4 & 18.6 & 8.3 & 10.1 & 11.6 & 37.3 & 1.7 & 61.0 & 100 \\
\hline East Kalimantan & 2.6 & 2.2 & 31.5 & 22.8 & 11.9 & 15.2 & 18.6 & 40.3 & 4.5 & 55.2 & 100 \\
\hline North Kalimantan & 2.8 & 2.5 & 35.5 & 25.4 & 13.2 & 18.4 & 7.6 & 41.6 & 7.4 & 51.0 & 100 \\
\hline Central Sulawesi & 2.2 & 2.4 & 27.2 & 27.0 & 21.7 & 16.4 & 7.6 & 34.8 & 1.6 & 63.6 & 100 \\
\hline Southeast Sulawesi & 2.8 & 2.8 & 39.7 & 22.9 & 16.7 & 13.4 & 7.3 & 52.4 & 0.7 & 46.9 & 100 \\
\hline Maluku & 3.1 & 2.9 & 39.1 & 26.2 & 17.6 & 12.9 & 4.2 & 54.8 & 1.6 & 43.6 & 100 \\
\hline North Maluku & 3.1 & 2.6 & 43.8 & 24.8 & 15.5 & 11.5 & 4.4 & 50.9 & 0.9 & 48.2 & 100 \\
\hline West Papua & 2.7 & 2.4 & 33.1 & 20.6 & 19.0 & 17.2 & 10.1 & 65.2 & 0.8 & 34.1 & 100 \\
\hline Papua & 2.9 & 2.0 & 46.6 & 17.1 & 10.6 & 14.0 & 11.7 & 71.6 & 1.7 & 26.6 & 100 \\
\hline R3 [Outside Java Bali 2] & 2.7 & 2.4 & 35.0 & 19.5 & 16.0 & 16.9 & 12.7 & 45.9 & 3.0 & 51.2 & 100 \\
\hline
\end{tabular}

Source: processed data.

Note: $\mathrm{CEB}=$ children ever born. 


\subsection{Logistic Model Analysis}

To generate regional-based models, the analysis looked at how unmet need correlates with selected socio-demographic variables and found that women aged 20-29 were more likely to experience an unmet need compared to their younger and older counterparts in all models (Table 4). Area of residence (urban vs rural) did not create a greater likelihood of unmet need but in model 1 (region 1/R1), husband's higher level of education did cause women to be 1.3 times less likely to use contraceptives. This situation was not observed in the other two models. In contrast, in all models, women's educational attainment had no significant predictive power of unmet need. The strongest predictor of unmet need was women's concept of ideal family size, as seen in model 3. Women who consider 2 or fewer children as an ideal family size were 1.4 times more likely to not use any contraception compared to women who desire a bigger family size $(\mathrm{OR}=1.439, \mathrm{CI}=1.173-1,767, \mathrm{P}=0.000)$. Similarly, as seen most markedly in model 3, women who have had 3 or more children were more likely to have an unmet need for family planning $(\mathrm{OR}=3,070$, $\mathrm{CI}=2,412-3,906, \mathrm{P}=0.000)$. Wealth quintile appears to have less predictive power of unmet need in region 3 as well as in other regions.

Logistic model analysis with regards to the 5 dimensions shows that ambivalent fertility preferences (D1) were particularly relevant in model 1 . Women who express a fatalistic view had a 2.7 higher probability to not adopt any family planning method compared to their counterpart $(\mathrm{OR}=2,768, \mathrm{CI}=2,104-3,641, \mathrm{P}=0.000)$. In model 2 and 3 , the probability was $1.8(\mathrm{OR}=1.804, \mathrm{CI}=1.193-2.729 \mathrm{P}=0.005)$ and $0.8 \quad(\mathrm{OR}=0,812, \mathrm{CI}=0,488-1,353, \mathrm{P}=0.812)$ respectively. Generic disapproval of pregnancy prevention (D2) was higher in model 1 (Java-Bali) and 3 (outside Java-Bali 2). Women who live in Java Bali had 1.6 (OR=1,627, CI=1,128-2,348, $\mathrm{P}=0.009)$ higher probability of not being able to use their preferred contraceptive method due to family and cultural opposition compared to women with no such social resistance. A similar higher probability of $1.4(\mathrm{OR}=1,476, \mathrm{CI}=0,810-2,689, \mathrm{P}=0.204)$ was found among women who live in region 3 (outside Java-Bali 2). In all models, method-specific barriers (D3) were found to have a very strong predictive power of unmet need especially in model 1 (Java-Bali) and 2 (outside Java-Bali 1). Women residing in these two regions had $4.3(\mathrm{OR}=4.363, \mathrm{CI}=4,404-7,280, \mathrm{P}=0.000)$ and $4.4(\mathrm{OR}: 4.451, \mathrm{CI}=3,705-5,347$, $\mathrm{P}=0,000)$ respectively greater odds of not protecting themselves from unintended pregnancy due to method-specific issues than women without those barriers. Women in region 3 (outside Java Bali 2) were 2.8 times (OR:2,808, $\mathrm{CI}=2,256-3,495, \mathrm{P}=0,000)$ more likely to decide to not use any modern contraceptive when they run into method-specific barriers. Contraceptive non-use as a result of women perceived low likelihood of becoming pregnant (D4) was most notably found in model 1 . In model 1, women who describe themselves as not likely to become pregnant were twice as likely as (OR:1,957, CI=1,707-2,243, $\mathrm{P}=0,000)$ fully aware women to not use any contraceptive. Similarly, women in the region outside Java Bali 1 who hold a similar perception were 1.4 times more likely (OR:1,446, CI=1,184-1,764, $\mathrm{P}=0,000)$ to be contraceptive non-users. Finally, the correlation between partner-related factors (D5) and unmet need for family planning was found to be negligible in all models. 
Table 4. Logistic Regression Model

\begin{tabular}{|c|c|c|c|c|c|c|c|c|c|c|c|c|c|c|c|}
\hline \multirow{3}{*}{ Variables } & \multicolumn{5}{|c|}{ Model 1 [Java Bali] } & \multicolumn{5}{|c|}{ Model 2 [Outside Java Bali 1] } & \multicolumn{5}{|c|}{ Model 3 [Outside Java Bali 2] } \\
\hline & \multirow{2}{*}{ B } & \multirow{2}{*}{ Sig. } & \multirow{2}{*}{$\operatorname{Exp}(B)$} & \multicolumn{2}{|c|}{$95 \% \mathrm{CI}$} & \multirow{2}{*}{$\mathbf{B}$} & \multirow{2}{*}{ Sig. } & \multirow{2}{*}{$\operatorname{Exp}(B)$} & \multicolumn{2}{|c|}{$95 \%$ CI } & \multirow{2}{*}{ B } & \multirow{2}{*}{ Sig. } & \multirow{2}{*}{$\operatorname{Exp}(B)$} & \multicolumn{2}{|c|}{$95 \%$ CI } \\
\hline & & & & $\mathbf{L L}$ & $\mathbf{U L}$ & & & & $\mathbf{L L}$ & $\mathbf{U L}$ & & & & $\mathbf{L L}$ & $\mathbf{U L}$ \\
\hline \multicolumn{16}{|l|}{ Urban [Ref] } \\
\hline Rural & 0,023 & 0,678 & 1,023 & 0,919 & 1,138 & 0,072 & 0,370 & 1,075 & 0,918 & 1,257 & $-0,013$ & 0,910 & 0,987 & 0,792 & 1,232 \\
\hline Age_Wife [Ref 45-49] & & 0,000 & & & & & 0,000 & & & & & 0,009 & & & \\
\hline Age_Wife [15-19] & 0,078 & 0,753 & 1,082 & 0,664 & 1,761 & 0,788 & 0,003 & 2,200 & 1,298 & 3,727 & 0,173 & 0,680 & 1,189 & 0,522 & 2,707 \\
\hline Age_Wife [20-24] & 0,382 & 0,000 & 1,465 & 1,189 & 1,805 & 0,939 & 0,000 & 2,557 & 1,862 & 3,510 & 0,385 & 0,084 & 1,470 & 0,950 & 2,274 \\
\hline Age_Wife [25-29] & 0,647 & 0,000 & 1,910 & 1,607 & 2,271 & 0,895 & 0,000 & 2,448 & 1,868 & 3,209 & 0,689 & 0,000 & 1,992 & 1,406 & 2,821 \\
\hline Age_Wife [30-34] & 0,291 & 0,001 & 1,338 & 1,131 & 1,582 & 0,703 & 0,000 & 2,019 & 1,567 & 2,603 & 0,365 & 0,030 & 1,440 & 1,036 & 2,001 \\
\hline Age_Wife [35-39] & 0,196 & 0,017 & 1,217 & 1,036 & 1,429 & 0,401 & 0,002 & 1,493 & 1,156 & 1,927 & 0,450 & 0,005 & 1,569 & 1,143 & 2,154 \\
\hline Age_Wife [40-44] & 0,208 & 0,009 & 1,231 & 1,053 & 1,439 & 0,593 & 0,000 & 1,809 & 1,415 & 2,312 & 0,222 & 0,173 & 1,249 & 0,907 & 1,720 \\
\hline $\begin{array}{l}\text { Level of Education_Husband } \\
{[\text { Ref_Low] }}\end{array}$ & & 0,000 & & & & & 0,908 & & & & & 0,770 & & & \\
\hline Level of Education_Husband [Middle] & 0,243 & 0,001 & 1,275 & 1,104 & 1,474 & $-0,015$ & 0,890 & 0,985 & 0,799 & 1,215 & 0,046 & 0,751 & 1,047 & 0,789 & 1,390 \\
\hline Level of Education_Husband [Above] & 0,315 & 0,000 & 1,371 & 1,182 & 1,590 & 0,030 & 0,776 & 1,031 & 0,838 & 1,267 & 0,100 & 0,470 & 1,105 & 0,843 & 1,449 \\
\hline Level of Education_Wife [Ref_Above] & & 0,079 & & & & & 0,011 & & & & & 0,137 & & & \\
\hline Level of Education_Wife_Low & 0,178 & 0,025 & 1,195 & 1,023 & 1,395 & $-0,275$ & 0,012 & 0,759 & 0,613 & 0,940 & $-0,255$ & 0,076 & 0,775 & 0,584 & 1,027 \\
\hline Level of Education_Wife_Middle & 0,066 & 0,340 & 1,069 & 0,932 & 1,225 & 0,020 & 0,847 & 1,020 & 0,835 & 1,246 & $-0,005$ & 0,972 & 0,995 & 0,758 & 1,307 \\
\hline \multicolumn{16}{|l|}{ Ideal Family Size [Ref_>=3] } \\
\hline Ideal Family Size [0-2] & 0,276 & 0,000 & 1,318 & 1,189 & 1,461 & 0,100 & 0,188 & 1,106 & 0,952 & 1,283 & 0,364 & 0,000 & 1,439 & 1,173 & 1,767 \\
\hline \multicolumn{16}{|l|}{ Children Ever Born [Ref_0-2] } \\
\hline Children Ever Born $[>=3]$ & 0,748 & 0,000 & 2,113 & 1,848 & 2,416 & 0,995 & 0,000 & 2,705 & 2,247 & 3,256 & 1,122 & 0,000 & 3,070 & 2,412 & 3,906 \\
\hline Wealth quintile_[Ref_Lowest] & & 0,010 & & & & & 0,770 & & & & & 0,760 & & & \\
\hline Wealth quintile_Middle Low & 0,163 & 0,088 & 1,176 & 0,976 & 1,418 & 0,027 & 0,799 & 1,027 & 0,837 & 1,260 & $-0,085$ & 0,544 & 0,919 & 0,699 & 1,208 \\
\hline Wealth quintile_Middle & 0,195 & 0,036 & 1,215 & 1,012 & 1,459 & 0,016 & 0,879 & 1,017 & 0,822 & 1,258 & $-0,203$ & 0,185 & 0,816 & 0,605 & 1,102 \\
\hline Wealth quintile_Middle_above & 0,063 & 0,504 & 1,065 & 0,886 & 1,280 & 0,114 & 0,336 & 1,120 & 0,889 & 1,413 & $-0,117$ & 0,443 & 0,889 & 0,659 & 1,200 \\
\hline
\end{tabular}




\begin{tabular}{|c|c|c|c|c|c|c|c|c|c|c|c|c|c|c|c|}
\hline Wealth quintile_Above & 0,262 & 0,006 & 1,299 & 1,077 & 1,568 & $-0,053$ & 0,703 & 0,949 & 0,724 & 1,244 & $-0,078$ & 0,651 & 0,925 & 0,660 & 1,296 \\
\hline $\begin{array}{l}\text { Weak or ambivalent fertility } \\
\text { preferences Dimension_Ref_No] }\end{array}$ & & & & & & & & & & & & & & & \\
\hline $\begin{array}{l}\text { Weak or ambivalent fertility preferences } \\
\text { Dimension [Yes] }\end{array}$ & 1,018 & 0,000 & 2,768 & 2,104 & 3,641 & 0,590 & 0,005 & 1,804 & 1,193 & 2,729 & $-0,208$ & 0,425 & 0,812 & 0,488 & 1,353 \\
\hline \multicolumn{16}{|l|}{$\begin{array}{l}\text { Generic disapproval of pregnancy } \\
\text { prevention [Ref_No] }\end{array}$} \\
\hline $\begin{array}{l}\text { Generic disapproval of pregnancy } \\
\text { prevention [Yes] }\end{array}$ & 0,487 & 0,009 & 1,627 & 1,128 & 2,348 & $-0,280$ & 0,329 & 0,756 & 0,430 & 1,326 & 0,389 & 0,204 & 1,476 & 0,810 & 2,689 \\
\hline \multicolumn{16}{|l|}{$\begin{array}{lllll}\text { Methods specific barriers } & \text { to } & \text { use } \\
\text { [Ref_No] } & & & & \end{array}$} \\
\hline Methods specific barriers to use [Yes] & 1,473 & 0,000 & 4,363 & 3,877 & 4,910 & 1,493 & 0,000 & 4,451 & 3,705 & 5,347 & 1,032 & 0,000 & 2,808 & 2,256 & 3,495 \\
\hline \multicolumn{16}{|l|}{$\begin{array}{l}\text { Perceived low risk of getting pregnant } \\
\text { [Ref_No] }\end{array}$} \\
\hline $\begin{array}{l}\text { Perceived low risk of getting pregnant } \\
\text { [Yes] }\end{array}$ & 0,671 & 0,000 & 1,957 & 1,707 & 2,243 & 0,368 & 0,000 & 1,446 & 1,184 & 1,764 & 0,026 & 0,857 & 1,027 & 0,771 & 1,368 \\
\hline \multicolumn{16}{|l|}{ Partner related factors_[Ref_No] } \\
\hline Partner related factors_Yes] & $-0,432$ & 0,017 & 0,649 & 0,456 & 0,925 & $-0,145$ & 0,565 & 0,865 & 0,528 & 1,418 & $-0,219$ & 0,436 & 0,803 & 0,463 & 1,393 \\
\hline Constant & $-2,311$ & 0,000 & 0,099 & & & $-2,098$ & 0,000 & 0,123 & & & $-1,944$ & 0,000 & 0,143 & & \\
\hline
\end{tabular}

Source: processed data

Note. $\mathrm{B}=$ beta; $\mathrm{Sig}=$ significant; $\exp =$ exponential $\mathrm{CI}=$ confidence interval; $\mathrm{LL}=$ lower limit; $\mathrm{UL}=$ upper limit 


\section{Discussion}

The International Conference on Population and Development (ICPD) Program of Action, the Sustainable Development Goals (SDGs) and Indonesia's Strategic Plan call for the acceleration of reducing the unmet need for family planning as part of the country's commitment to achieve universal health coverage. This indicator is viewed as an instrument to promote maternal and child health, family planning and reproductive health in Indonesia's 2020-2024 National Medium-Term Development Plan, which was officially established through Presidential Regulation No.18/2020. Indonesia has set a target of reducing unmet need to $7.4 \%$ by 2024 and successful achievement of the target will depend on appropriate strategies and tailored interventions that recognize different sub-groups and territory segmentations. This viewpoint stems from the insight that unmet need varies across regions due to different cultural and socio-demographic variables that shape the reasons for unmet need (Population Reference Bureau, 2012).

Levels of unmet need in the three regions partly reflect the respective region's family planning program maturity. The percentage of unmet need is highest in R3 where family planning was introduced at a later stage than in the other two regions. Uneven distribution of healthcare providers across provinces also play a role, and there is a larger concentration of healthcare providers in the Java Bali region than in other regions (Statistics Indonesia, 2020). In addition, despite some common characteristics, women with unmet need for family planning in Indonesia are quite unique. Unlike other studies that found unmet need as more associated with younger, less-educated women in a rural setting who have less-educated husband and have fulfilled their fertility intention, Indonesian women with unmet need in all regions are actually the opposite (Juarez, Gayet \& Meija-Pailles, 2018; Bhattathiry \& Ethiranjan, 2014; Asif, M \& Pervaiz, 2019). A higher percentage of women with unmet need live in urban areas. This finding is consistent with the 2017 Indonesian Demographic and Health Survey unmet need data of $11 \%$ in urban area vs $10 \%$ in rural area. IDHS also reported that there is greater reliance on traditional methods in the urban area than in rural areas ( 8 percent vs. 4.9 percent). Nevertheless, in all three regions, no significant correlation was seen between prevalence of unmet need and region of residence. The urban-rural dichotomy that is often linked to the risk of unmet need is less relevant in Indonesia, primarily due to massive family planning program implementation at the village level through the "Kampung Keluarga Berencana" (family planning village) initiative and high-level political commitment (Endah \& Kholid, 2019).

The increasing percentage of unmet need with older age, larger ideal family size, and number of children ever born, suggests a more prominent unmet need for limiting rather than for spacing births (Klijzing, 2000; Hameed, Azmat, Bilgrami \& Ishaqe, 2011). The likelihood of unmet need with respect to age forms an inverted-U configuration in model 1 and 2, but fluctuates in model 3. Generally, the highest probability of unmet need was found among women aged 20-29, which is contradictory to a previous study that reported that the odds for unmet need were higher for young adolescents between the age of 15-19 compared with women of advanced reproductive age (Lamichhane, 2017). The reason was because young adolescents are already sexually active, would like to postpone pregnancy, yet experience bigger barriers to access contraceptive. In the Indonesian context, the association of unmet need with the 20-29 age group seems to be related with women's median age at first marriage, which is 20.8 (National Population and Family Planning Board et al., 2018). This is when they commonly start to need contraception. For women living in R3, number of children and ideal family size are better predictors of unmet need, more so than for women in the other two regions.

It is worth noting that spouse's educational level has a stronger predictive power than the wife's, particularly in model 1. This finding is less consistent with other research that indicate how less educated women are more likely to endure unmet need compared to women with some level of education (Bhusal \& Bhattarai, 2018, Ali \& Okud, 2013; Hailemariam \& Haddis, 2011). This study also does not support a study from Nepal where better educated women are more likely to have unmet need due to an expansion of information on reproductive health in schools allowing a growing demand for family planning but yet inaccessible services. Findings about unmet need and husband's educational level are a reverse of what was reported from Eastern Sudan where less educated husbands were associated with an almost 2 times greater risk of unmet need than better educated husbands (Ali \& Okud, 2013). Our study found that husbands who attain a higher level of education in the Java Bali region have a higher probability of experiencing unmet need. This is thought to be due to their better knowledge about the adverse effects of contraceptives on their wife's health, leading them to opt for traditional methods.

This study also did not find a strong association between unmet need and economic status. This might imply that there is little out-of-pocket payment because family planning service has been incorporated into the national health insurance scheme since 2014. The premium for the poor is paid by the central and local government for short as well as long acting and permanent methods, unlike in Nepal (Lamichhane, 2017) or Madhya Pradesh 
(Sahasrabuddhe, Kori, Arora \& Bute, 2018) where family planning services are more accessible to the rich than to low-income women.

The awareness and knowledge of contraceptives is best presented in the Java Bali region (model 1) where the likelihood of unmet need relative to D1, D2 and D4 is highest than in other regions. The study confirms that the fatalistic outlook of women does shape contraceptive use in Indonesia, which is most noticeable in model 1. Ambivalent fertility preferences discourage women from accessing contraceptives even though they do not want to get pregnant, contrary to findings that women's belief in God's role in pregnancy has no well-defined relationship with their fertility behavior (Jones, Frohwirth \& Blades, 2016). Such view does not inhibit some women from accessing contraceptives and they might in turn use them consistently. In this study, the 'fate' or 'destiny' notion is more likely to function as a remedy to liberate women from having unintended pregnancies.

It is widely accepted that myths and misconceptions about family planning are a barrier to adoption of modern contraceptive methods. Abundant studies have shown how various myths about contraception, uncomfortable of side effects, cultivation of misinformation at the community level, and association between contraception and promiscuity, straying, and infertility have prevented women from using contraception (Sedlander et al., 2018; Yang et al., 2015; Ochako et al., 2015). This is an issue that deserves close attention as these non-user women in Indonesia have little chance to discuss family planning when they visit a health facility (National Population and Family Planning Board et. al, 2018). Generic disapproval may also be caused by religious prohibition as noted among Muslim women in India who have less future intention to use contraceptives (Patra \& Singh, 2015). However, in Indonesia the legality of family planning has been well established since 1974 in the Rabat Conference on family planning. It reaffirmed that no verse or text in the Quran explicitly prohibits a wife or husband to participate in family planning. Having children is encouraged in the Islamic perspective but at the same time, each family has a duty to fully fulfill their obligations towards the increasing demand of raising children (Sadik et al., 1992).

A recent study revealed that unmet need in Indonesia, as in many other developing countries, is mainly caused by existing pain points in the family planning program. Insufficient provision of information and counseling regarding family planning have caused women to simply not use contraceptives, discontinue current methods or switch to traditional methods (Cavallaro et al.,2019). Self-reported reasons associated with method-specific barriers emerge as the most influential factor of unmet need, particularly in model 1 and 2. Knowledge of contraceptive methods is nearly universal in Indonesia. Almost all currently married women aged 15-49 have heard of at least one modern method (National Population \& Family Planning Board et al., 2018). However, a gap remains between having knowledge and having the necessary information to voluntarily adopt and sustain contraceptive use. Studies found that only 62 percent of Indonesian women received information about contraceptive choices from their health or family planning field workers, only 44 percent were told about possible side effects and only one third were informed about what they need to do upon experiencing any side effects (National Population \& Family Planning Board et al.,2018). Furthermore, a third of Indonesian women (33 percent) cited fear of side effects as their reason to discontinue their current contraceptive methods (National Population \& Family Planning Board et al., 2018). Even though Indonesia has implemented universal health coverage as mentioned earlier, critical challenges in providing equitable access, ensuring quality services and financial protection for people still persist (Ardiana, Ekoriano \& Fathonah, 2019). Similar findings about universal health coverage from the health perspective have been reported from Thailand and even Europe (Reeves, Mckee \& Stuckler, 2015; Yan et al., 2020).

Women's perception of being at little risk of becoming pregnant and subsequent decision to not use any modern contraceptive might jeopardize their reproductive goal. A rigorous study in 57 countries in 2015 showed that 62 percent of women have unmet need right after delivery, while 43 and 32 percent of women have unmet need after six months of amenorrhea and at the end of the amenorrhea period respectively (Rossier, Bradley, Ross \& Winfey, 2015). Breastfeeding women are less likely to be using contraception because they are much less likely to expect another child than a woman who had discontinued breastfeeding (Machiyama \& Cleland, 2013).

Spousal separation entails severely different coital frequency. It leads to almost two times higher proportion of unmet need among women whose husbands stay at home than that among women whose husbands are away for more than 3 months in 40 rural districts in Nepal (Ban, Karki, Shrestha \& Hodgins, 2012). A couple whose husband is temporarily living elsewhere does not necessarily mean that they do not require contraception but their needs might differ from couples who live together.

The association between husband's disapproval and possibility of unmet need appears to be insignificant in all regions. It is consistent with the 2017 Indonesian Demographic and Health Survey result that 38 percent of women 
make their own decision about contraceptive method, and having their husbands make the decision is less likely (National Population \& Family Planning Board et al., 2018). This finding disapproves another finding from Saudi Arabia indicating that one fourth of unmet need is a result of husbands' opposition (Khalil, Alzahrani \& Siddiqui, 2017).

\subsection{Limitation of the Study}

Data of the study limits the analysis only for national and provincial levels. It prevents a valuable insight of policy makers at districts level to formulate appropriate policy response in their respective juridictions. According to the adopted model of unmet need, the calculation considers both for modern and traditional methods. Currently, Indonesia is struggling to improve contraceptive method mix by promoting long acting and permanent methods as it is more effective to protect couples from unwanted pregnancy than traditional ones and at the same time the short- term methods indicate high discontinuation rate among pill and injectable users. It also appears that traditional method users are those perceiving their methods as a choice rather than a condition for not being able to access services as they are better educated, wealthier and mostly live in the urban area (National Population and Family Planning Board et al., 2018).

\section{Conclusion}

This study emphasizes the uneven trajectory of unmet need within Indonesia. It is sensitive to the maturity of the family planning program as well as socio-economic factors. This study provides insights for policymakers to better formulate appropriate policies and effectively utilize available resources, while continue to close the gap between different geographic areas. For research purposes, it is highly recommended that any questionnaire used to identify the underlying factors of unmet need be equipped with more specific description and incorporate more precise program performance monitoring indicators. Further research is also needed to better understand the uniqueness of Indonesia's findings, particularly those that are contrary to generally-accepted results from other countries. In the longer term, there is a call to do more research and development for more contraceptive options with fewer side effects.

\section{Acknowledgements}

The authors would like to express their sincere gratitude to the National Population and Family Planning Board (BKKBN) especially to Hasto Wardoyo, the Chairman of BKKBN for his valuable guidance. High appreciation is given to Prof. Rizal Damanik, Deputy of Training, Research and Development, BKKBN for his fruitful discussion. The authors would like also to acknowledge the contribution of Robert Ainslie, Senior Technical Advisor, John Hopkins Center for Communication Program in Indonesia for his valuable review of the paper.

\section{Competing Interests Statement}

The authors declare that there are no competing or potential conflicts of interest.

\section{References}

Asif, M. F., \& Pervaiz, Z. (2019). Socio-demographic determinants of unmet need for family planning among married women in Pakistan. BMC Public Health, 19(1), 1226. https://doi.org/10.1186/s12889-019-7487-5

Ali, A. A. A., \& Okud, A. (2013). Factors affecting unmet need for family planning in Eastern Sudan. BMC Public Health, 13(1), 102. https://doi.org/10.1186/1471-2458-13-102

Ardiana, I., Ekoriano, M., \& Fathonah, S. (2019). Universal health coverage 2019 in Indonesia: The integration of family planning services in current functioning health system. Journal of Population and Social Studies. https://doi.org/10.25133/JPSSv27n3.0016

Ban, B., Karki, S., Shrestha, A., \& Hodgins, S. (2012). Spousal separation and interpretation of contraceptive use and unmet need in Rural Nepal. International Perspectives on Sexual and Reproductive Health, 38(1), 43-47. https://doi.org/10.1363/3804311

Bishwajit, G., Tang, S., Yaya, S., \& Feng, Z. (2017). Unmet need for contraception and its association with unintended pregnancy in Bangladesh. BMC Pregnancy and Childbirth, 17(1), 1-9. https://doi.org/10.1186/s12884-017-1379-4

Bradley, S. E. K., Croft, T. N., \& Fishel, J. D. (2012). Revising unmet need for family planning: DHS analytical studies no. 25 (Issue January). USA: ICF International. https://dhsprogram.com/pubs/pdf/AS25/AS25\%5B12June2012\%5D.pdf

Bellizzi, S., Mannava, P., Nagai, M., \& Sobel, H. L. (2020). Reasons for discontinuation of contraception among women with a current unintended pregnancy in 36 low and middle-income countries. Contraception, 101(1), 
26-33. https://doi.org/10.1016/j.contraception.2019.09.006

Bhattathiry, M., \& Ethirajan, N. (2014). Unmet need for family planning among married women of reproductive age group in urban Tamil Nadu. Journal of Family and Community Medicine, 21(1), 53. https://doi.org/10.4103/2230-8229.128786

Bhusal, C. K., \& Bhattarai, S. (2018). Factors affecting unmet need of family planning among married Tharu women of Dang District, Nepal. International Journal of Reproductive Medicine, 2018, 1-9. https://doi.org/10.1155/2018/9312687

Cavallaro, F. L., Benova, L., Owolabi, O. O., \& Ali, M. (2020). A systematic review of the effectiveness of counselling strategies for modern contraceptive methods: What works and what doesn't? BMJ Sexual and Reproductive Health, 46(4), 254-269. https://doi.org/10.1136/bmjsrh-2019-200377

Endah, K., \& Kholiq, A. W. (2019). Pelaksanaan program kampung keluarga berencana oleh Dinas Keluarga Berencana, Pemberdayaan Perempuan dan Perlindungan Anak dalam mewujudkan kehidupan masyarakat sejahtera: Studi Desa Babakan, Kecamatan Pangandaran, Kabupaten Pangandaran [Implementation of the family planning program by the family planning office for women's empowerment and child protection in realizing a prosperous community life: A study from Babakan village, Pangandaran sub-district, Pangandaran district]. Jurnal Moderat, $\quad$ 5(2), 101-112. https://jurnal.unigal.ac.id/index.php/moderat/article/view/2402/2099

Hameed, W., Azmat, S. K., Bilgrami, M., \& Ishaqe, M. (2011). Determining the factors associated with unmet need for family planning: A crosssectional survey in 49 districts of Pakistan. Pakistan Journal of Public Health, 1(1), 21-27. Retrieved from https://www.researchgate.net/publication/236942649_Determining_the_factors_associated_with_Unmet_ne ed_for_family_planning_a_cross-sectional_survey_in_49_districts_of_Pakistan

Hailemariam, A., \& Haddis, F. (2011). Factors affecting unmet need for family planning in southern nations, nationalities and peoples region, Ethiopia. Ethiopian Journal of Health Sciences, 21(2), 77-89. https://doi.org/10.4314/ejhs.v21i2.69048

Juarez, F., Gayet, C., \& Mejia-Pailles, G. (2018). Factors associated with unmet need for contraception in Mexico: Evidence from the National Survey of Demographic Dynamics 2014. BMC Public Health. https://doi.org/10.1186/s12889-018-5439-0

Jones, R. K., Frohwirth, L. F., \& Blades, N. M. (2016). "If i know I am on the pill and i get pregnant, it's an act of God": Women's views on fatalism, agency and pregnancy. Contraception, 93(6), 551-555. https://doi.org/10.1016/j.contraception.2016.02.005

Klijzing, E. (2000). Are there unmet family planning needs in Europe? Family Planning Perspectives, 32(2), 74-81, 88. https://doi.org/10.1363/3207400

Khalil, S. N., Alzahrani, M. M., \& Siddiqui, A. F. (2018). Unmet need and demand for family planning among married women of Abha, Aseer Region in Saudi Arabia. Middle East Fertility Society Journal, 23(1), 31-36. https://doi.org/10.1016/j.mefs.2017.07.004

Lamichhane, K. (2017). Unmet need for family planning among currently married young women in Nepal. Journal of Development and Administrative Studies, 25(1-2), 83-94. https://doi.org/10.3126/jodas.v25i1-2.23446

Machiyama, K., \& Cleland, J. (2013). Insights into unmet need in Senegal. STEP UP research report. London: London School of Hygiene \& Tropical Medicine. Retrieved from https://www.popcouncil.org/uploads/pdfs/2013RH_STEPUPUnmetNeed_Senegal.pdf

Machiyama, K., Casterline, J. B., Mumah, J. N., Huda, F. A., Obare, F., Odwe, G., .. \& Cleland, J. (2017). Reasons for unmet need for family planning, with attention to the measurement of fertility preferences: Protocol for a multi-site cohort study. Reproductive Health, 14(1), 23. https://doi.org/10.1186/s12978-016-0268-z

Ministry of National Development Planning/National Development Planning Agency. (2020). Roadmap of SDGs Indonesia towards 2030.Indonesia, Jakarta. Retrieved from http://sdgs.bappenas.go.id/dokumen/

National Population and Family Planning Board. (2018). Survei kinerja dan akuntabilitas program 2018 [Government performance and accountability program survey 2018]. Jakarta, Indonesia. Retrieved from https://cis.bkkbn.go.id/latbang/?p=15 
National Population and Family Planning Board. (2019). Laporan kinerja Badan Kependudukan dan Keluarga Berencana Nasional 2018 [Accountability report of National Population and Family Planning Board 2018]. Jakarta, Indonesia.

National Population and Family Planning Board. (2020). Rencana strategis Badan Kependudukan dan Keluarga Berencana Nasional tahun 2020-2024 [Strategic plan of National Population and Family Planning Board year 2020-2024]. Jakarta, Indonesia.

National Population and Family Planning Board (BKKBN), Statistics Indonesia (BPS), Ministry of Health (Kemenkes), and ICF. (2018). Indonesia demographic and health survey 2017. Jakarta, Indonesia: BKKBN, BPS, Kemenkes, and ICF Retrieved from https://dhsprogram.com/pubs/pdf/FR342/FR342.pdf

Ochako, R., Mbondo, M., Aloo, S., Kaimenyi, S., Thompson, R., Temmerman, M., \& Kays, M. (2015). Barriers to modern contraceptive methods uptake among young women in Kenya: A qualitative study. BMC Public Health, 15(1), 1-9. https://doi.org/10.1186/s12889-015-1483-1

Sadik, N., Omran, A. R. (Ed.), \& Sjadzali, H. (1992). Family planning in the legacy of Islam. London: Routledge, https://doi.org/10.4324/9780203167977

Patra, S., \& Singh, R. K. (2015). Addressing unmet need and religious barrier towards the use of family planning method among Muslim women in India. International Journal of Human Rights in Healthcare, 8(1), 22-35. https://doi.org/10.1108/IJHRH-06-2014-0010

Population Reference Bureau. (2012). Unmet need for family planning. Population Reference Bureau. Retrieved from https://www.prb.org/unmet-need/

Reeves, A., McKee, M., \& Stuckler, D. (2015). The attack on universal health coverage in Europe: Recession, austerity and unmet needs. European Journal of Public Health, 25(3), 364-365. https://doi.org/10.1093/eurpub/ckv040

Rossier, C., Bradley, S. E., Ross, J., \& Winfrey, W. (2015). Reassessing unmet need for family planning in the postpartum period. Studies in Family Planning, 46(4), 355-367. https://doi.org/10.1111/j.1728-4465.2015.00037.x

Sedlander, E., Bingenheimer, J. B., Thiongo, M., Gichangi, P., Rimal, R. N., Edberg, M., \& Munar, W. (2018). "They destroy the reproductive system": Exploring the belief that modern contraceptive use causes infertility. Studies in Family Planning, 49(4), 345-365. https://doi.org/10.1111/sifp.12076

Soesastra, H., Budiman, A., Triaswati, N., Alisjahbana, A., \& Adiningsih, S. (2005). Pemikiran dan pemasaran ekonomi di Indonesia dalam setengah abad terakhir: Buku 3 (1966-1982) Paruh pertama ekonomi orde baru [Economic thought and marketing in Indonesia in the last half century: Book 3 (1966-1982) First half of the new order economy]. Kanisius publishing \& Ikatan Sarjana Ekonomi Indonesia (ISEI). DI. Yoyakarta. Retrieved from https:/www.goodreads.com/book/show/6403241-pemikiran-dan-permasalahan-ekonomi-di-indonesia-dala m-setengah-abad-tera

Statistics Indonesia. (2020). Doctor distribution in Indonesia in 2019. Retrieved from https://databoks.katadata.co.id/datapublish/2020/03/26/persebaran-dokter-di-indonesia-2019

Sahasrabuddhe, A., Kori, S., Arora, V. K., \& Bute, J. (2018). A study to assess unmet need for family planning and contraceptive choices among married women of reproductive age in rural Madhya Pradesh. International Journal of Community Medicine and Public Health, 5(11), 4725. https://doi.org/10.18203/2394-6040.ijcmph20184207

WHO. (2019). High rates of unintended pregnancies linked to gaps in family planning services: New WHO study. Retrieved from https:/www.who.int/news-room/detail/25-10-2019-high-rates-of-unintended-pregnancies-linked-to-gaps-infamily-planning-services-new-who-study

Yan, L. D., Hanvoravongchai, P., Aekplakorn, W., Chariyalertsak, S., Kessomboon, P., Assanangkornchai, S., ... \& Stokes, A. C. (2020). Universal coverage but unmet need: National and regional estimates of attrition across the diabetes care continuum in Thailand. PLoS ONE, 15(1), 1-14. https://doi.org/10.1371/journal.pone.0226286

Yang, G., Sau, C., Lai, W., Cichon, J., \& Li, W. (2015). Belief in family planning myths at the individual and community levels and modern contraceptive use in urban Africa. Int Perspect Sex Reprod Health, 344(6188), 
1173-1178. https://doi.org/10.1363/4119115.Belief

\section{Appendix. The distribution of provinces by dimension of unmet need}
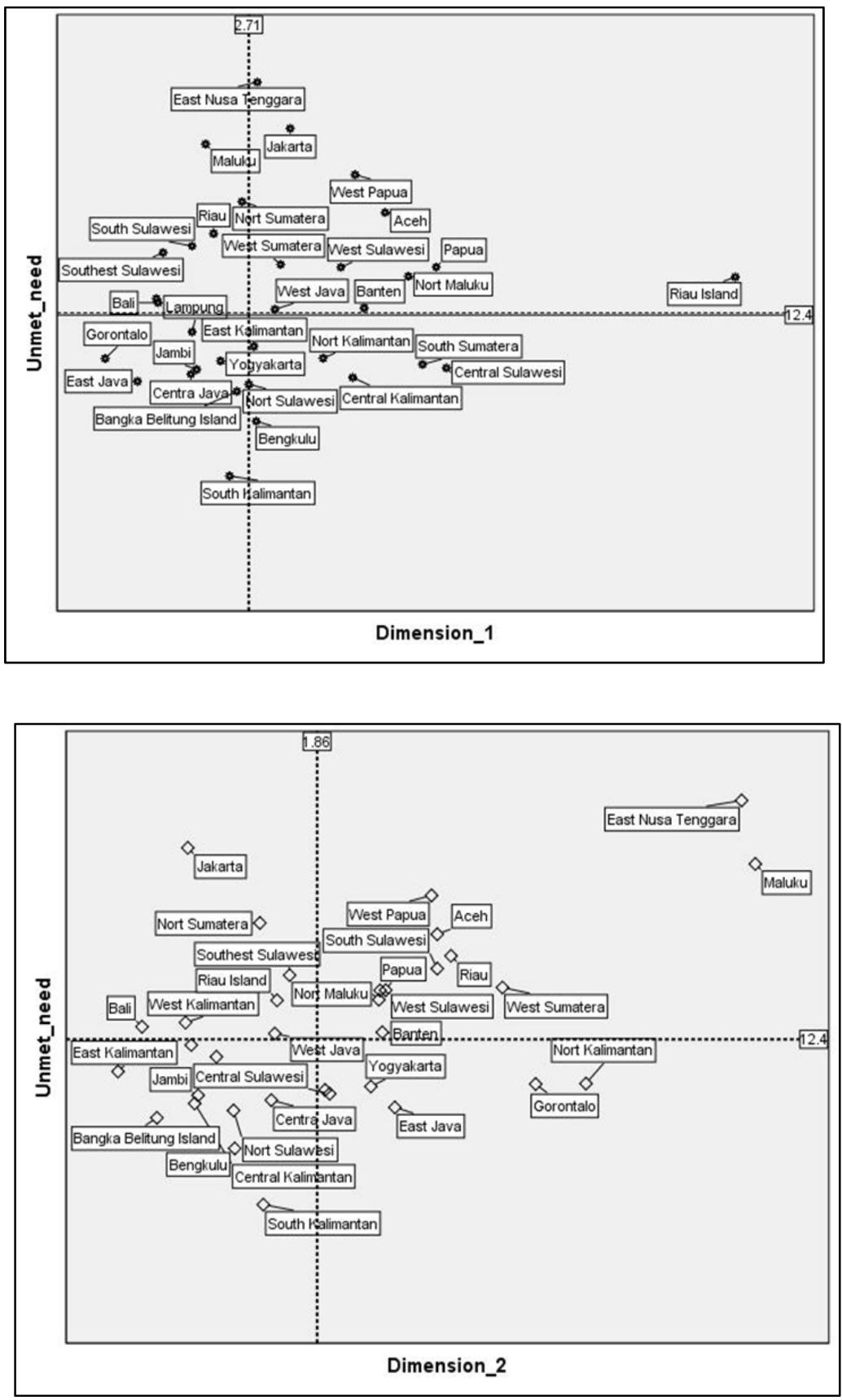

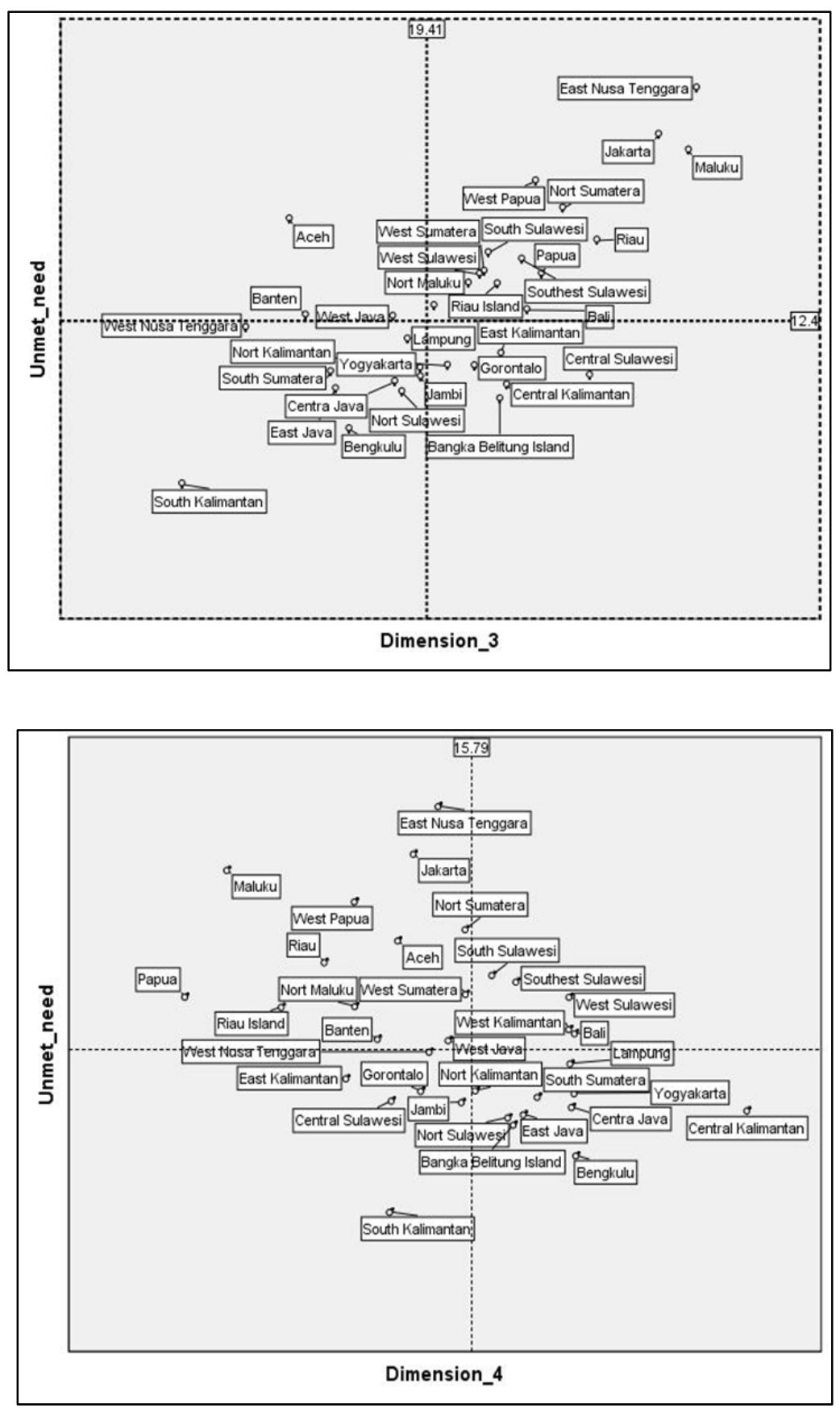


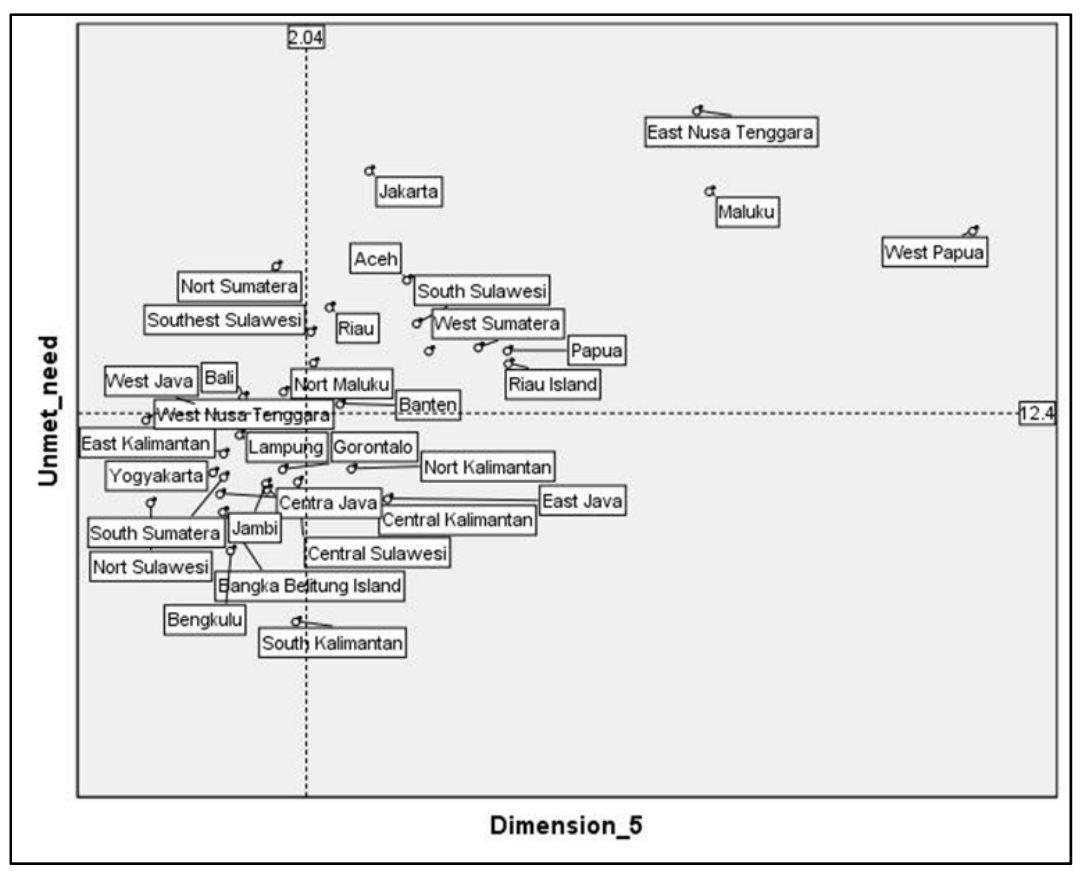

\section{Copyrights}

Copyright for this article is retained by the author(s), with first publication rights granted to the journal.

This is an open-access article distributed under the terms and conditions of the Creative Commons Attribution license (http://creativecommons.org/licenses/by/4.0/). 